Parents scaffold flexible imitation in early childhood

Jennifer M. Clegga

Cristine H. Legare ${ }^{a}$

${ }^{\mathrm{a}}$ The University of Texas at Austin, Department of Psychology

1 University Station \#A8000, Austin, TX 78712, USA

Correspondence concerning this article should be addressed to Jennifer M. Clegg, The University of Texas at Austin, Department of Psychology, 1 University Station \#A8000, Austin, TX 78712, (512) 232-1289. Email: jclegg@utexas.edu. The raw data associated with the current research can be found at www.cristinelegare.com. 


\begin{abstract}
Children use imitation flexibly to acquire the instrumental skills and conventions of their social groups. This study ( $N=69$ parent and 3-6-year-old child dyads) examined the impact of instrumental versus conventional language on (a) children's imitative flexibility in the context of parent-child interaction and (b) how parents scaffold children's imitation. Children in dyads presented with conventional language imitated with higher fidelity than children in dyads presented with instrumental language. Parents in dyads presented with conventional language also provided their children with more instruction to imitate and engaged in more encouragement, demonstration, and monitoring than parents in dyads presented with instrumental language. The relation between language cue and children's imitative fidelity was mediated by parent scaffolding behavior. The results provide evidence that caregivers support the development of flexible imitation in early childhood by adjusting their scaffolding according to the goal of the behavior.

keywords: conventions; flexible imitation; instrumental skills; parent-child interaction; scaffolding; socialization
\end{abstract}




\section{Parents scaffold flexible imitation in early childhood}

Children are highly selective about the types of behaviors they imitate and to what degree, discriminating between when they need to learn a process or reproduce an outcome. Though much of the research on children's imitation has focused on children's use of high fidelity imitation, or overimitation, as a social learning strategy to acquire instrumental skills (Horner \& Whiten, 2005; Lyons, Damrosch, Lin, Macris, \& Keil, 2011), recent work suggests that accounts of overimitation place too much emphasis the role of children's causal reasoning in imitation (Legare \& Nielsen, 2015). Children certainly do imitate instrumental behaviors to gain objectrelated, causal knowledge (DiYanni, Nini, \& Rheel, 2011; Lyons, Young, \& Keil, 2007; McGuigan, Whiten, Flynn, \& Horner, 2007; Nielsen, 2006), but much of what children learn using imitation is not based in causal reasoning, but in social conventionality (Kenward, Karlsson, \& Persson, 2010; Nielsen \& Blank, 2011; Nielsen, Simcock, \& Jenkins, 2008; Over \& Carpenter, 2012). Children also use imitation to acquire the conventional behaviors (Diesendruck \& Markson, 2011; Keupp, Behne, \& Rakoczy, 2013; Over \& Carpenter, 2012) or rituals of their social groups (Legare \& Watson-Jones, 2015; Watson-Jones \& Legare, 2016; Wen, Herrmann, \& Legare, 2015). To be efficient cultural learners, children must use imitation flexibly to acquire both instrumental skills and the conventions of their social groups (Legare \& Nielsen, 2015).

Flexible imitation is driven by the interpretation of the goal of a behavior as an instrumental versus a conventional act (Legare, Wen, Herrmann, \& Whitehouse, 2015). The objectives of imitating instrumental and conventional behaviors are distinct. The objective of imitating instrumental behavior is reproducing the end-goal by discerning which actions are causally relevant to producing the desired outcome (Clegg \& Legare, 2016a; Herrmann, Legare, Harris, \& Whitehouse, 2013; Nielsen, Kapitány, \& Elkins, 2015). Attending to the causal 
relationship between the actions and the end-goal allows for innovation and variability in the reproduction of the behavior (Carr, Kendal, \& Flynn, 2015), and, as a result, lower fidelity imitation. In contrast, the objective of imitating conventional behavior is reproducing the process (Call, Carpenter, \& Tomasello, 2005; Schachner \& Carey, 2013), which requires attending to the way the behavior ought to be executed (Haun, Rekers \& Tomasello, 2012; Kallgren, Reno, \& Cialdini, 2000; Kapitány \& Nielsen, 2015; Over \& Carpenter, 2013; Rakoczy, Warneken, \& Tomasello, 2008). Thus, in contrast to imitating instrumental behaviors, imitating conventional behaviors requires consistently high fidelity imitation (Legare et al., 2015). Recent studies have demonstrated that the high fidelity imitation of conventional behavior may be socially-motivated or driven by the desire to engage in social group affiliation (Kenward et al., 2010; Keupp, et al., 2013; Nielsen, Cucchiaro, \& Mohamedally, 2012; Over \& Carpenter, 2009; Watson-Jones, Whitehouse, \& Legare, 2015).

Children interpret a behavior as an instrumental act if the physical-causal basis of the actions is potentially knowable and relevant to the efficient execution of the behavior. In contrast, children interpret a behavior as a conventional act if the actions are based on social stipulation rather than physical causality (Legare et al., 2015). Much of our behavior, however, is not easily interpretable as instrumental or conventional based on observation alone. For example, hand washing can incorporate instrumental elements (e.g., wetting hands) and conventional elements (e.g. using a particular vessel to hold water) and can have an instrumental goal (e.g., removing pathogens) or a conventional goal (e.g., a purification ritual). A child observing hand washing behavior must determine whether she should engage in high fidelity imitation of the process (e.g., rinsing her hands a specific number of times, saying a particular phrase during the process) or whether she should eliminate elements that are not causally relevant for achieving the end-goal. 
In both situations, the child must use social cues to determine whether a behavior has an instrumental or conventional goal. Much of our behavior includes both instrumental and conventional elements, thus inferences about the goal of a behavior are often a matter of degree rather than kind (Legare \& Nielsen, 2015; Legare et al., 2015).

How do children determine whether a behavior is instrumental or conventional? Children are sensitive to a number of social and contextual cues when making inferences about the goal of behavior, including causal opacity (i.e., action sequences with identical start- and end-states or observable non-functional components), consensus (i.e., multiple actors performing the same actions), and synchrony (i.e., multiple actors performing the same actions at the same time (DiYanni, Corriveau, Kurkul, Nasrini, \& Nini, 2015; Herrmann et al., 2013; Legare et al., 2015; Watson-Jones, Legare, Whitehouse, \& Clegg, 2014), and verbal cues (Clegg \& Legare, 2016a; 2016b). In the current study we build upon previous research examining the impact of verbal cues to instrumental versus conventional behavior on imitative flexibility in early childhood (Clegg \& Legare, 2016a).

Young children's imitative fidelity becomes increasingly specialized during early childhood. For example, there are age-related improvements in object-memory based imitation between the ages of 2- and 5-years-old (Subiaul, Patterson, Schilder, Renner, \& Barr, 2014). In addition, children discriminate between instrumental and social cues at 4-years-old but not at 2years-old (Yu \& Kushnir, 2013). Children's understanding of the social and contextual cues used to distinguish between instrumental and conventional behavior also increases with age. Older children demonstrate a greater difference in imitative fidelity between instrumental and conventional tasks than younger children (Clegg \& Legare, 2016a; Legare et al., 2015). Children may become more sensitive to these cues over the course of ontogeny due to learning about 
social conventionality (Diesendruck \& Markson, 2011; Josephs, Kushnir, Gräfenhain, \& Rakoczy, 2016; Köymen et al., 2014; Rakoczy \& Schmidt, 2013).

How does imitative flexibility develop? Understanding the development of imitative flexibility requires understanding socialization. Caregivers and children are collaborators in children's social learning (Callanan, Siegel, \& Luce, 2007; Lave \& Wegner, 1991; Rogoff, 2003). Children not only appear to have a preference for learning from adults (Harris, 2012; Jaswal \& Neely, 2006; VanderBorght \& Jaswal, 2009), they may also learn more in activities in which they are interacting with parents than when interacting with peers (Bjorklund, Hubertz, \& Reubens, 2004; Gauvain, 2001; Radziszewska \& Rogoff, 1989; 1991). This may be due to parents' pedagogical skill in providing assistance to their children and scaffolding their children's behavior. Given the active role of caregivers in children's social development (Callanan \& Jipson, 2001), understanding the ontological emergence flexible imitation requires examining the extent to which caregivers scaffold different levels of imitative fidelity for instrumental versus conventional behaviors.

\section{1. The Present Study}

To assess children's imitative flexibility in the context of parent-child interaction, we presented parent-child dyads with a necklace-making task with both instrumental (i.e., bead stringing) and conventional elements (i.e., bead shape and color choice, novel gestures). Clegg and Legare (2016a, 2016b) have demonstrated that in the standard experimenter-child design, children who hear a conventional framing (i.e., "Everyone always does it like this.") before watching a demonstration of the necklace-making task imitate with higher fidelity than children who hear an instrumental framing (i.e., "I'm going to make a necklace."). They also found that this difference in imitative fidelity between the conditions was due to children hearing 
conventional language rather than children receiving instructions to copy, indicating that the difference in children's imitative fidelity between conditions were not simply due to the demand characteristics of the task.

Here we build upon previous work examining children's imitative flexibility in the context of experimenter-child settings (see Legare \& Nielsen, 2015 for a review), by investigating children's imitative fidelity in the context of parent-child interaction. We predicted that, like in experimenter-child settings, children in parent-child dyads who hear conventional language would imitate with higher fidelity than children in dyads who hear instrumental language. To assess the influence of parents' behavior on children's imitative fidelity, we also examined the proportion of imitative actions that children engaged in as a result of parent instruction. We predicted that a greater proportion of children's imitative fidelity would be the result of parent instruction for dyads in the conventional condition than the instrumental condition.

Our primary objective was to examine the kinds of behavior that parents use to socialize and scaffold children's imitative flexibility. In particular, we measured parents' scaffolding behaviors: encouragement of children's imitation of the model, demonstration of particular target actions, and monitoring of their children's behaviors. We coded both parents' verbal encouragement and their demonstration of the actions they saw the research assistant model. We predicted that parents would be more likely to generally reference the modeled example and to encourage a demonstrated action by name or demonstrate the action themselves in the conventional condition in order to encourage high fidelity imitation. In addition to parents' encouragement and demonstration, we examined parent monitoring, or whether or not parents verbally or physically stopped their children from completing an action while working on 
completing their necklace. Parent monitoring of child behavior indicates parental supervision and enforcement of the goal of the task. We predicted that parents would engage in more monitoring of child behavior in the conventional condition. In sum, we predicted that parents presented with conventional language would (a) engage in more verbal encouragement and demonstration of the modeled actions and (b) demonstrate higher levels of monitoring, than parents presented with instrumental language.

We were also interested in the impact that these scaffolding behaviors might have on children's imitative fidelity. While in this study, we did not directly compare solo children to children in parent-child pairs, we were able to assess the direct impact of these behaviors on children's imitation through mediation analyses. We predicted that the relationship between language cue (instrumental versus conventional) and children's imitative fidelity would be mediated by the scaffolding behaviors we measured.

To assess how parent-child interaction might change over the course of early childhood development, we included 3- to 6-year-old children and one of their parents in the study. We predicted that dyads with older children would engage in higher levels of imitative fidelity given previous research indicating that older children imitate with higher fidelity than younger children (Clegg \& Legare, 2016a; Herrmann et al., 2013; Lyons, et al., 2007; McGuigan, et al., 2007; Nielsen \& Tomaselli, 2010; Watson-Jones et al., 2014; Yu \& Kushnir, 2013). We also predicted that parents would engage in more scaffolding of younger than older children, consistent with previous research on age-differences in parent-child interaction (Pèrez-Grandos \& Callanan, 1997; Rogoff, Ellis, \& Gardner, 1984).

\section{Method}

\section{1. Participants}


Sixty-nine parent-child dyads (49 mothers, 32 female children, mean child age $=4.83$ ) were recruited from a children's science museum in an urban university town in the American southwest. Children were between the ages of 3- and 6-years-old. The families recruited were primarily Euro-American and from middle- to high-socioeconomic status families. Data from 5 additional dyads were dropped due to sibling interference (3), or the pair using a language other than English during the necklace-making task (2).

\section{2. Procedure}

Testing was conducted in a quiet room in the children's museum. During the experiment the parent and child sat across a table from the experimenter. All dyads in the study participated in an imitation task and were randomly assigned to one of two conditions (instrumental and conventional conditions) in a between-subjects design. During this task, the parents and children watched an experimenter demonstrate a necklace-construction sequence and then were given an opportunity to interact with the stimuli. Before beginning each study session, the experimenter explained to the parent that none of the tasks were tests and that the parent and child should act as they normally would when playing a game together.

\section{3. Imitation task}

After the parent-child dyad completed a warm-up game to allow them to become more relaxed and familiar with the study's setting, the experimenter told them that they would be completing a new activity. The experimenter also informed the parent and child that after her demonstration, she would leave the room to allow them to complete the activity together. After this, she placed a set of necklace-making materials (a metal tray with one row of 3 round beads purple, orange, and green (left to right from the experimenter's perspective) - in front of a row of 3 cube beads - red, yellow, and blue (left to right) in front of two folded strings - red and green) 
on the table. The experimenter then told the dyad one of two language prompts while smiling, with both hands flat on either side of the tray: In the instrumental condition, pairs heard an outcome-oriented explanation of the task (e.g. "I am going to make a necklace. Let's watch what I am doing. I am going to make a necklace.”). In the conventional condition pairs heard a convention-oriented explanation (e.g. "Everyone always does it like this. Let's watch what I am doing. Everyone always does it like this.").

The experimenter began the sequence by looking down and picking up the red string. She held one end of the red string in each hand, stretched the string into a straight line, and then brought the ends back together in front of her. The experimenter repeated this action once more before stretching the string into a straight line and placing it in front of the tray (the side closest to the child) and removing both of her hands. The experimenter then picked up the purple round bead and touched it to her forehead before stringing it on the right side of the string and moving the bead to the middle of the string. The experimenter repeated this sequence for the yellow square bead and the green round bead. After the experimenter placed the green bead on the string, she picked one end of the string up in each hand, held the necklace up, and while smiling said, "Look what I did!" For an illustration of the action sequence, see Figure 1. After finishing the sequence, the experimenter placed the necklace back on the tray and removed the tray from the child's view. Dyads in both conditions viewed the same sequence of actions.

After the task demonstration, the experimenter gave the dyad a duplicate set of the beads and string, positioned in the same way (left to right). While the experimenter moved the tray of objects toward the dyad she said, "Now it's your turn! Here you go." After the experimenter gave the dyad the tray, she left the room in order to remove the social influence of her presence on the dyad's interactions and waited for the parent or child to indicate that they had completed 
the activity. Each dyad's engagement with the objects was video-recorded and coded for imitative fidelity, different parental scaffolding behaviors, and whether the dyad was engaging in parallel or coordinated activities. The imitation task was based on a necklace-making task developed by Clegg \& Legare (2016a).

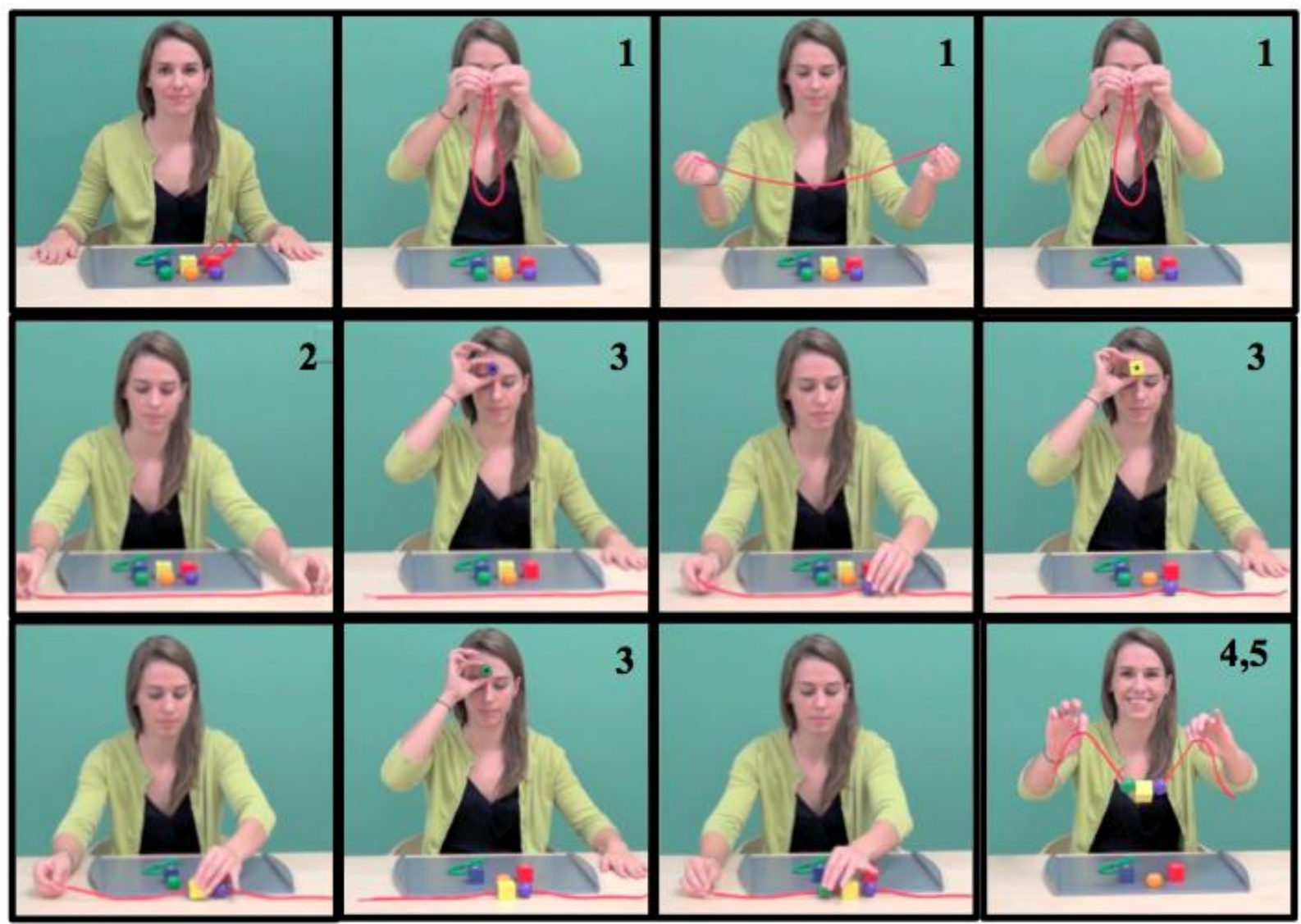

\section{Component of action sequence}

1. Stretch string

2. Place string

3. Bead to forehead touch

4. Circle, square, circle

5. Three beads
Operational criteria for coding

The child brings the ends of the string together and opens it at least once.

The child lays the string out on the table and removes both hands.

The child touches at least one bead to their forehead before placing it on the string.

The child's necklace consists of a circular bead, a square bead, and a circular bead in order.

The child's necklace consists of only three beads.

Figure 1. Necklace-making sequence and target elements included in imitative fidelity score. 


\section{4. Measures}

A primary coder blind to condition and the hypotheses of the study coded each measure.

Data from $40 \%$ of the sample (28 children) was independently coded to assess inter-rater reliability. The second coder was blind to the hypotheses of the study and the condition to which each child was assigned. Reliability is reported for each measure.

2. 4. 1. Imitative fidelity score. Each dyad was given a summary score between 0 and 5 based on the number of target actions of the modeled necklace-making sequence the child imitated (the table in Figure 1 illustrates scoring categories; Clegg \& Legare, 2016a). If the pair made more than one necklace, only the first necklace that the child contributed to was coded. Coders demonstrated 94\% agreement with the Kappa (.89) for this coding falling within very good agreement (.81 and above) levels (Landis \& Koch, 1977).

2. 4. 1. 1. Instructed imitative fidelity. Children were coded for whether they completed each of the target actions included in the imitative fidelity score independently or directly following instruction from their parent. Instruction was defined as the parent either verbally encouraging or demonstrating the behavior immediately before the child complied and engaged in the behavior. Each child was given an instructed imitative fidelity proportion that was calculated by dividing the number of target actions that the child completed after instruction by their total imitative fidelity score. Coders demonstrated $92 \%$ agreement with the Kappa (.84) for this coding falling within very good agreement (.81 and above) levels (Landis \& Koch, 1977). 2. 4. 2. Parent scaffolding. Parents were coded for whether or not they engaged in different scaffolding behaviors, including encouragement, demonstration of target actions, and monitoring.

2. 4. 2. 1. Encouragement. Encouragement was defined as the parent verbally instructing, prompting, or asking a child about behavior related to the necklace-making task. Encouragement 
was coded for two categories: encouragement that referred to the model (model action) and encouragement to engage in one of the five target actions included in the imitative fidelity score (target action). For a description of the different encouragement content categories, see Table 1. We coded for model action encouragement to capture those instances when parents referred to the model, but not necessarily a specific action. We interpret parent encouragement that referred to the model, even generally, as the parents' attempt to guide their children's behavior to complete the task as it was demonstrated and engage in high fidelity imitation. Reliability was calculated for both parent encouragement categories and coders demonstrated $100 \%$ agreement.

2. 4. 2. 2. Demonstration of target action. Parents were coded for whether they demonstrated one of the five target actions included in the imitative fidelity score. Demonstration was defined as the parents engaging in one of the target actions while orienting their attention toward their child, rather than engaging in the action in parallel play with the child. See Table 1 for examples. Reliability was calculated for the parent demonstration categories and coders demonstrated $100 \%$ agreement.

2. 4. 2. 3. Monitoring. Each pair was coded for whether or not the parent verbally or physically stopped their child from completing an action at least while working on completing the task. See Table 1 for examples. Reliability was calculated for the parent demonstration categories and coders demonstrated $100 \%$ agreement. 
Table 1.

Descriptions and examples of parent scaffolding categories.

\begin{tabular}{|c|c|c|}
\hline Category & Description & Examples \\
\hline $\begin{array}{l}\text { Encouragement - } \\
\text { Model action }\end{array}$ & $\begin{array}{l}\text { When the parent encourages the } \\
\text { child to do or think of what the } \\
\text { model did without referring to a } \\
\text { specific action. }\end{array}$ & $\begin{array}{l}\text { "Do you remember what she did?" } \\
\text { "Let's try to make it like she did." }\end{array}$ \\
\hline $\begin{array}{l}\text { Encouragement - } \\
\text { Target action }\end{array}$ & $\begin{array}{l}\text { Parent encourages child to engage } \\
\text { in at least one of the five target } \\
\text { actions: stretch string, place } \\
\text { string, bead to forehead touch, } \\
\text { making a circle, square, circle } \\
\text { pattern, or using three beads. }\end{array}$ & $\begin{array}{l}\text { "Can you move the string like she did?" } \\
\text { "Can you put the string on the table?" } \\
\text { "Touch it to your head." } \\
\text { "We need two circles and a square." } \\
\text { "How many beads did she use?" }\end{array}$ \\
\hline Demonstration & $\begin{array}{l}\text { Parent engages in one of the five } \\
\text { target actions included in the } \\
\text { imitative fidelity score while } \\
\text { orienting their attention toward } \\
\text { their child, rather than engaging in } \\
\text { the action in parallel play with the } \\
\text { child. }\end{array}$ & $\begin{array}{l}\text { Parent places bead on forehead while } \\
\text { drawing attention to self and looking at } \\
\text { child. } \\
\text { Parent picks up string and brings the ends } \\
\text { of the string together and opens it at least } \\
\text { once. }\end{array}$ \\
\hline Monitoring & $\begin{array}{l}\text { Parent verbally or physically stops } \\
\text { their child from completing an } \\
\text { action at least while working on } \\
\text { completing the task. }\end{array}$ & $\begin{array}{l}\text { Child is about to string bead and adult says, } \\
\text { "No, first touch it to your head." } \\
\text { Child reaches for a bead and adult } \\
\text { physically stops the child's hand before it } \\
\text { can reach the bead. }\end{array}$ \\
\hline
\end{tabular}

\section{Results}

Parent and child sex were included as factors in preliminary analyses for all measures and were not found to have significant effect and was removed from subsequent analyses. Multiple regression analyses were conducted to examine the effects of condition (instrumental, conventional) and child age (coded as a continuous variable) on each of the following measures unless otherwise noted. There was not a significant interaction between condition and child age for any of the analyses so these interactions were removed. 


\section{1. Imitative fidelity}

Condition and child age accounted for a significant amount of the variability in imitative fidelity, $R^{2}=.16, F(2,66)=6.17, p=.004$ (Figure 2 ). As predicted, children engaged in higher levels of imitative fidelity in the conventional condition $(M=3.21, S D=1.47)$ than in the instrumental condition $\left(M=2.34, S D=1.06 ; \beta=0.90, p=.004\right.$, partial $\left.\eta^{2}=.14\right)$. Imitative fidelity also increased with child age $\left(\beta=0.28, p=.047\right.$, partial $\left.\eta^{2}=.06\right)$. Thus, the results indicated that older children in the conventional condition had the highest levels of imitative fidelity.

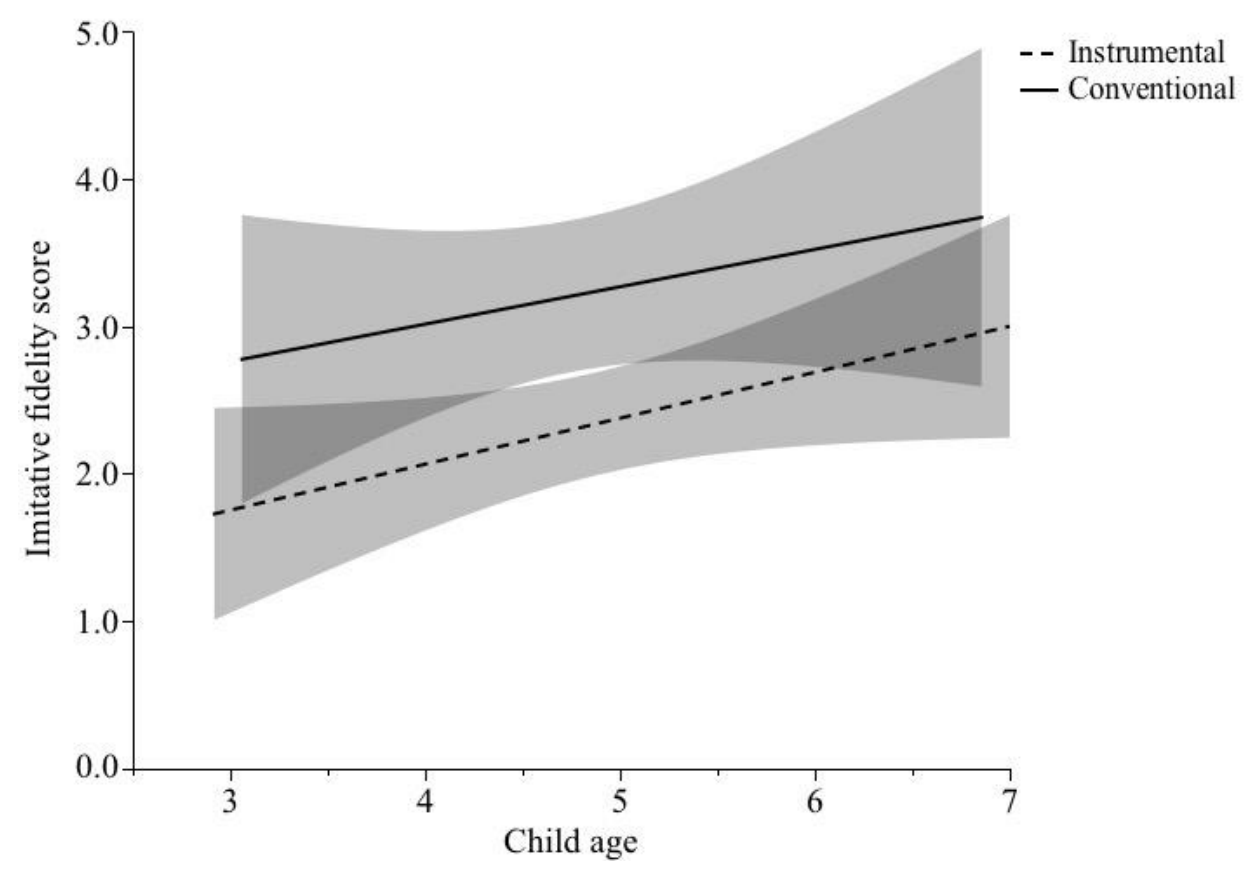

Figure 2. Relationship between age and imitative fidelity score by condition. Shaded area indicates $95 \%$ confidence of fit for each regression line.

3. 1. 1. Instructed imitative fidelity. Condition and child age accounted for a significant amount of the variability in the proportion of each pair's imitative fidelity score that was completed after instruction, $R^{2}=.11, F(2,66)=4.27, p=.018$. As predicted, a greater proportion of children's imitative fidelity scores were instructed in the conventional condition $(M=0.17$, 
$S D=0.26)$ than in the instrumental condition $\left(M=0.07, S D=0.19 ; \beta=0.12, p=.032\right.$, partial $\eta^{2}$ $=.06)$. The proportion of each pair's imitative fidelity score that was the result of instruction also increased with child age $\left(\beta=0.05, p=.042\right.$, partial $\left.\eta^{2}=.07\right)$. Older children in the conventional condition had the highest proportion of actions that were completed after instruction in their imitative fidelity scores.

\section{2. Parent scaffolding}

Binary logistic regressions were performed to examine the effects of condition and child age on whether parents engaged in each of the scaffolding measures (encouragement - model action, encouragement - target action, demonstration of target action, and monitoring). See Figure 3 for the percent of parents engaging in each of the scaffolding measures by condition. There was not a significant interaction between condition and child age for any of the analyses so these interactions were removed.

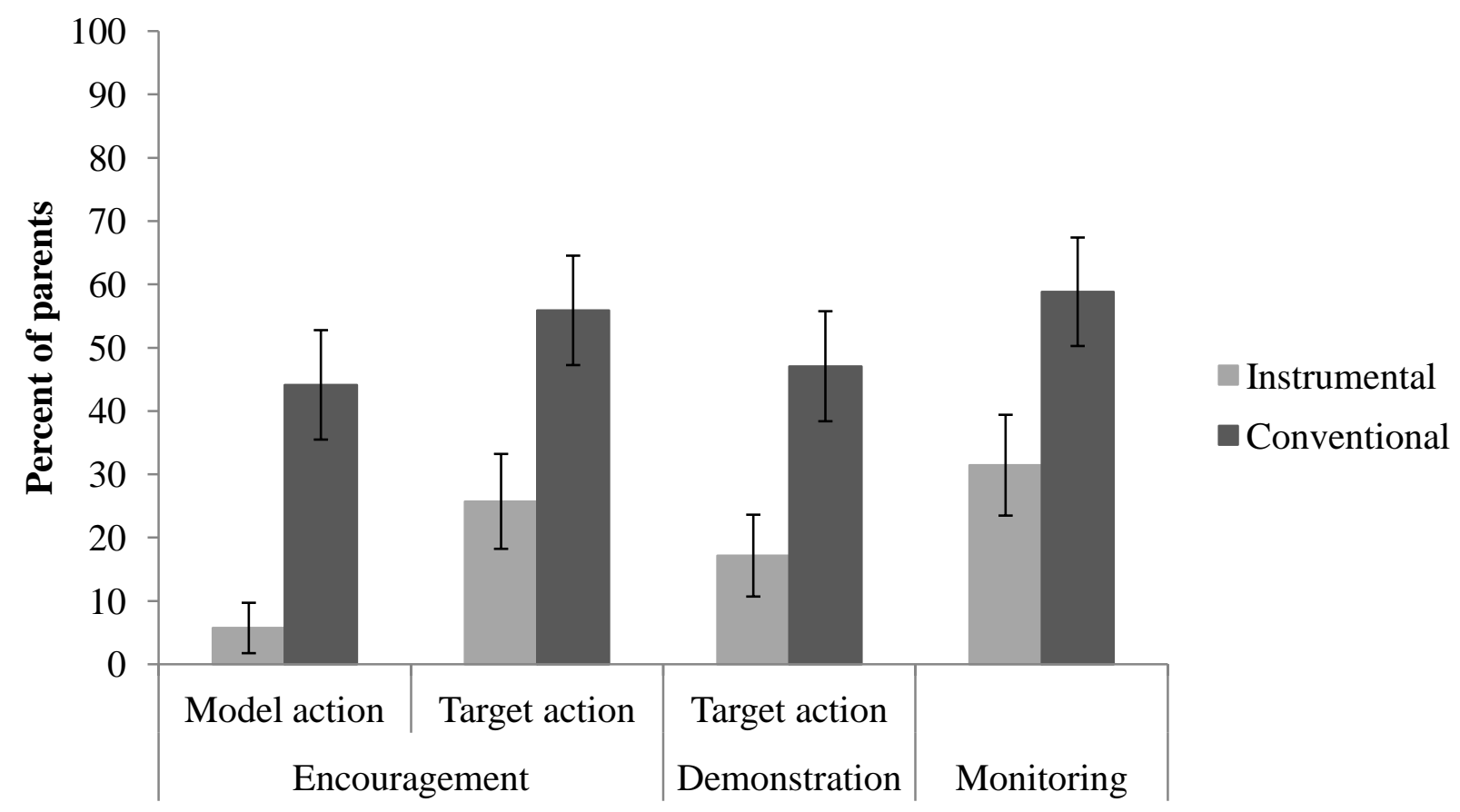

Figure 3. Percent of parents engaging in scaffolding measures by condition. Error bars represent 1 SEM. 


\section{2. 1. Encouragement.}

3. 2. 1. 1. Model action. The results of the analysis indicate that condition did have a significant impact on whether parents engaged in model action encouragement ( $p$ $<.001)$, but age did not $(p=.824)$. Parents in the conventional condition $(44.1 \%)$ were more likely to engage in encouragement that generally referenced the model than parents in the instrumental condition $(5.71 \%$; odds ratio $=3.41)$.

3. 2. 1. 2. Target action. The results of the analysis indicate that condition did have a significant impact on whether parents engaged in target action encouragement ( $p$ $=.028)$, but age did not $(p=.279)$. Parents in the conventional condition (55.9\%) were more likely to engage in encouragement that specifically referenced one of the modeled target actions than parents in the instrumental condition $(25.7 \%$; odds ratio $=2.03)$.

3. 2. 2. Demonstration of target action. The results of the analysis indicate that condition did have a significant impact on whether parents demonstrated at least one of the target actions $(p=.009)$, but age did not $(p=.374)$. Parents in the conventional condition $(47.1 \%)$ were more likely to demonstrate at least one of the target actions than parents in the instrumental condition $(17.1 \% ;$ odds ratio $=2.37)$.

3. 2. 3. Monitoring. The results of the analysis indicate that condition did have a significant impact on whether parents engaged in monitoring $(p=.001)$, but age did not $(p$ $=.330)$. Parents in the conventional condition $(58.8 \%)$ were more likely to engage in monitoring of their children's actions than parents in the instrumental condition $(31.4 \%$; odds ratio $=3.01)$. 


\section{3. Mediation analysis}

We used a path analysis to test the hypothesis that the measured parent scaffolding behaviors mediated the effect of condition on children's imitative fidelity. We included each of the behaviors (encouragement - model action, encouragement - target action, demonstration, and monitoring) individually in the model to assess the indirect effect of each (e.g., condition $\rightarrow$ scaffolding behavior $\rightarrow$ imitative fidelity). We controlled for age when building both the indirect effects and the direct effect (e.g., condition $\rightarrow$ imitative fidelity). The results of the mediation test are presented in Figure 4. As Figure 4 illustrates, the standardized regression coefficients between condition and each of the scaffolding behaviors were all significant, but the only significant standardized regression coefficient between the scaffolding behaviors and imitative fidelity was for monitoring. When controlling for all four of the scaffolding behaviors and age, condition was no longer a significant predictor of children's imitative fidelity, $b=0.145, S E=$ $0.336, p=.262$, consistent with full mediation. Approximately $20 \%$ of the variance in children's imitative fidelity was accounted for by the parent scaffolding behaviors, with monitoring accounting for approximately $12 \%$ alone. 


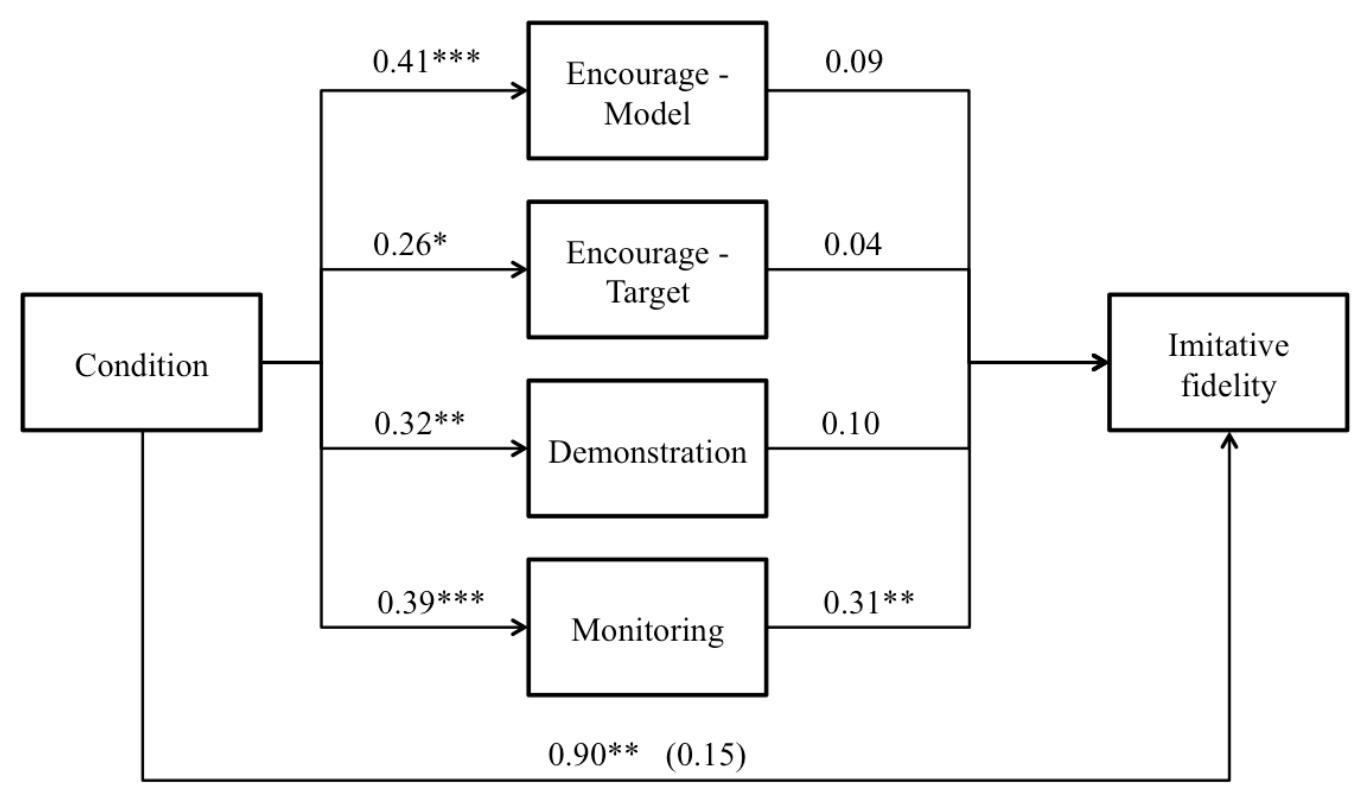

Figure 4. Standardized regression coefficients for the relationship between condition and children's imitative fidelity as mediated by parent scaffolding behaviors, controlling for child age. The standardized regression coefficient between condition and imitative fidelity, controlling for parent scaffolding behaviors, is in parentheses.

$* p<.05, * * p<.01, * * * p<.001$

\section{Discussion}

Children's ability to engage in flexible imitation of instrumental versus conventional actions is reflective of the ways caregivers reinforce and scaffold imitative behavior. The data from this study indicate that language cues to instrumental and conventional goals for behaviors impact both children's imitative fidelity in the context of parent-child interaction and parents' interactions with their children. In addition, parents' scaffolding behaviors mediate the relation between the language cues and children's imitative fidelity.

Children in dyads in the conventional condition engaged in higher levels of imitative fidelity than children in dyads in the instrumental condition. Our data are consistent with and directly comparable to a study completed with children acting independently (Clegg \& Legare, 
2016a) and suggest that parents supported and facilitated children's decisions to engage in high versus low imitative fidelity as a result of the language cue presented before the task. Differences in children's imitative fidelity between conditions in this study do not exclusively reflect parental sensitivity to the differences between the cues given that children show differences in imitative fidelity based on cues to instrumental and conventional behavior on their own (Clegg \& Legare, 2016a; 2016b). Yet data from a number of measures, including the instructed imitative fidelity measure and the parent scaffolding measures, indicate that parents structure children's imitative fidelity in different ways based on their interpretation of the task as instrumental or conventional. Moreover, the parent scaffolding behaviors mediate the relation between language cue and children's imitative fidelity.

The data from the instructed imitative fidelity measure provide the first indication that parents scaffold the difference in children's imitative fidelity between conditions. A greater proportion of children's imitative fidelity scores was the immediate result of parental instruction for dyads in the conventional condition. This suggests that parents were instructing their children to engage in higher imitative fidelity and children responded to their parents' instruction more in the conventional condition than in the instrumental condition.

There is evidence that a greater proportion of older children's (versus younger children's) imitative fidelity scores were the result of parent instruction, however, we interpret this cautiously given the main effect of age found for imitative fidelity scores across conditions. Consistent with previous research, older children had higher imitative fidelity scores overall than younger children, possibly due to improvements in memory and attention (Clegg \& Legare, 2016a; Herrmann et al., 2013; Legare et al., 2015; Subiaul \& Schidler, 2014). Because older children had greater imitative fidelity scores, there were more behaviors that could have been the 
result of parental instruction. Previous research has demonstrated that parents engage in higher amounts of scaffolding for younger children (Pèrez-Grandos \& Callanan, 1997; Rogoff et al., 1984). Yet our data showed no effects of child age on whether or not parents engaged in the specific verbal encouragement and demonstration behaviors we coded for. Thus, the main effect of age for instructed imitative fidelity may not reflect a difference in parent behaviors as children get older, but rather an improvement in children's ability to or interest in responding to parent instruction over the course of development.

Furthermore, the parent scaffolding data are consistent with our proposal that parents' behaviors reflect their interpretation of the goal of the modeled behavior. Parents interacted with their children in distinct ways between conditions. For example, parents in the conventional condition were more likely to use verbal encouragement that referred to the model and to specifically encourage one or more of the target actions of the modeled necklace-making sequence. Not only were parents verbally encouraging their children to refer to the model's demonstration and to engage in specific actions more in the conventional condition, they were also more likely to demonstrate the modeled actions for their children. In addition to scaffolding children's high fidelity imitation through encouragement and demonstrations, parents in the conventional condition were also more likely to monitor their children's behavior - stopping their children before they completed an action. This same level of monitoring was not seen in the instrumental condition, which is consistent with a situation where the parent and child were both engaged in the activity of making a necklace, rather than the parent closely watching and correcting the child so that she engaged in a specific process. Together, these data provide evidence that parents who interpret the goal of a task to be conventional are more likely to scaffold their children's high fidelity imitation. Parents who interpret the goal of a task as 
instrumental do not engage in the same level of scaffolding of their children's behaviors, ostensibly because they are not attempting to encourage high fidelity imitation, but rather the execution of an instrumental goal.

These differences in scaffolding behaviors between conditions had an impact on children's imitative fidelity - the relation between language cue and children's imitative fidelity was significantly mediated by the indirect path via parent scaffolding behaviors. This suggests that in the context of parent-child interaction, the scaffolding behaviors parents engaged in in response to the language cues influenced children's imitative flexibility. Specifically, whether or not parents engaged in monitoring had a significant impact on children's imitative fidelity. Whereas the other scaffolding behaviors might have helped to remind children of the types of actions they needed to engage in without directly leading to a change in behavior, monitoring might have had a particular impact because it, by design, stopped children from engaging in particular behaviors that ostensibly did not relate to executing the demonstrated process.

There is mounting evidence for cultural variation in child socialization (Legare \& Harris, 2016). Research indicates that didactic pedagogy in dyadic parent-child interaction is more common in WEIRD (i.e., Western, Educated, Industrialized, Rich, Democratic) cultures (Henrich, Heine, \& Norenzeyan, 2010; Little, Carver, \& Legare, 2016), and that Western childrearing privileges child-directed interaction and instruction from adults (Callaghan et al., 2011; Lancy, 2010; 2015). In contrast, non-Western childrearing emphasizes children's observational learning rather than direct instruction (Gaskins \& Paradise, 2010; Lancy, 2016; Rogoff, 2003). Cross-cultural differences in the kinds of information children attend to when learning new information and behaviors have also been documented, such that children from more collectivist backgrounds are more likely to defer to majority (Corriveau \& Harris, 2010) 
and consensus (Corriveau, Kim, Song, \& Harris, 2013; DiYanni et al., 2015). Given cultural variation in child socialization, future research is needed to examine continuity and variation in how caregivers scaffold imitative flexibility. We predict that caregivers in Western cultural contexts would engage in higher levels of direct instruction, whereas caregivers in Non-Western cultural contexts might engage more in demonstrative behaviors (e.g., completing the task as modeled) without ostensive verbal instruction and monitoring or appeal more to consensus information (e.g. "This is how everyone does it.") to encourage children to imitate. Moreover, children in non-Western cultural contexts also tend to spend more time in peer-based activity, so it is also important to examine how children imitate in peer-based settings (López, CorreaChávez, Rogoff, \& Gutiérrez, 2010; Nielsen, Mushin, Tomaselli, \& Whiten, 2016). Future studies can also examine caregiver-child interaction during more naturalistic conventional versus instrumental tasks, such as engaging in a purification ritual versus hand washing as we suggest in the introduction.

Taken together, our data provide convergent evidence that caregivers support the development of flexible imitation in early childhood by adjusting their interactions with their children according to the goal of the behavior and differentially socialize instrumental versus conventional imitation. These findings give novel insight into the kinds of scaffolding caregivers engage in to guide their children's flexible imitation, a crucial prerequisite for efficient social learning. By gaining insight into how caregivers socialize flexible imitation in early childhood, we can better understand how children come to distinguish between conventional and instrumental behavior and use this distinction to guide their imitation. 


\author{
Author note: \\ This research was supported by a National Science Foundation Graduate Research Fellowship \\ Grant to the first author and an ESRC (Economic and Social Research Council) Large Grant \\ [REF RES-060-25-0085] to the second author. Special thanks to the Thinkery and its staff for \\ facilitating data collection. Thank you to Paige E. Hartman and Christine Chevis for their help \\ with data collection. We also thank Colin Bannard and Aiyana K. Willard for their statistical \\ insight and analysis advice.
}




\section{References}

Bjorklund, D., Hubertz, M., \& Reubens, A. (2004). Young children's arithmetic strategies in social context: How parents contribute to children's strategy development while playing games. International Journal of Behavioral Development, 28(4), 347-357. doi: $10.1080 / 01650250444000027$

Call, J., Carpenter, M., \& Tomasello, M. (2005). Copying results and copying actions in the process of social learning: chimpanzees (Pan troglodytes) and human children (Homo sapiens). Animal Cognition, 8, 151-163. doi: 10.1007/s10071-004-0237-8

Callaghan, T., Moll, H., Rakoczy, H., Warneken, F., Liszkowski, U., Behne, T., \& Tomasello, M. (2011). Early Social Cognition in Three Cultural Contexts. Monographs of the Society for Research in Child Development, 76(2), 1-142.

Callanan, M. A., \& Jipson, J. L. (2001). Explanatory conversation and young children's developing scientific literacy. In K. Crowley, C.D. Schumm, and T. Okada (Eds.), Designing for science: Implications from everyday, classroom, and professional settings. Mahwah, NJ: Lawrence Erlbaum Associates.

Callanan, M. A., Siegel, D., \& Luce, M. R. (2007). Conventionality in family conversations about everyday objects. In M. Sabbagh \& C. Kalish (Eds.), New Directions for Child and Adolescent Development (pp. 83-97). San Francisco: Jossey-Bass.

Carr, K., Kendal, R. L., \& Flynn, E. G. (2015). Imitate or innovate? Children's innovation is influenced by the efficacy of observed behaviour. Cognition, 42, 322-332. doi: 10.1016/j.cognition.2015.05.005 
Clegg, J.M., \& Legare, C.H. (2016a). Instrumental and conventional interpretations of behavior are associated with distinct outcomes in early childhood. Child Development, 87(2), 527-542. doi: 10.1111/cdev.12472

Clegg, J.M. \& Legare, C.H. (2016b, in press). A cross-cultural comparison of children's imitative flexibility. Developmental Psychology.

Corriveau, K. H., \& Harris, P. L. (2010). Preschoolers (sometimes) defer to the majority in making simple perceptual judgments. Developmental Psychology, 46(2), 437. doi: $10.1037 / \mathrm{a} 0017553$

Corriveau, K. H., Kim, E., Song, G., \& Harris, P. L. (2013). Young children's deference to a consensus varies by culture and judgment setting. Journal of Cognition and Culture, 13(3-4), 367-381. doi: 10.1163/15685373-12342099

Diesendruck, G., \& Markson, L. (2011). Children's assumption of the conventionality of culture. Child Development Perspectives, 5(3), 189-195. doi: 10.1111/j.1750-8606.2010.00156.x

DiYanni, C.J., Corriveau, K. H., Kurkul, K., Nasrini, J., \& Nini, D. (2015). The role of consensus and culture in children's imitation of inefficient actions. Journal of Experimental Child Psychology, 137, 99-110. doi:10.1016/j.jecp.2015.04.004

DiYanni, C.J., Nini, D., \& Rheel, W. (2011). Looking good versus doing good: Which factors take precedence when children learn about new tools? Journal of Experimental Child Psychology, 110(4), 575-591. doi: 10.1016/j.jecp.2011.06.002

Gaskins, S. and Paradise, R. (2010) Learning through observation. In D.F., Lancy, J. Bock, \& S. Gaskins (Eds.), The anthropology of learning and childhood (pp. 85-117). Lanham, MD: Alta Mira Press. 
Gauvain, M. (2001). The social context of cognitive development. New York: Guilford Press.

Harris, P. L. (2012). Trusting what you're told: How children learn from others. Cambridge, MA: Harvard University Press.

Haun, D. B., Rekers, Y., \& Tomasello, M. (2012). Majority-biased transmission in chimpanzees and human children, but not orangutans. Current Biology, 22, 727-731.

doi: 10.1016/j.cub.2012.03.006

Henrich, J., Heine, S. J., \& Norenzayan, A. (2010). Most people are not WEIRD. Nature, 466, 29-29. doi: 10.1038/466029a

Herrmann, P.A., Legare, C.H., Harris, P.L., \& Whitehouse, H. (2013). Stick to the script: The effect of witnessing multiple actors on children's imitation. Cognition, 129, 536-543. doi: 10.1016/j.cognition.2013.08.010

Horner, V., \& Whiten, A. (2005). Causal knowledge and imitation/emulation switching in chimpanzees (Pan troglodytes) and children (Homo sapiens). Animal Cognition, 8(3), 164-181. doi: 10.1007/s10071-004-0239-6

Jaswal, V. K., \& Neely, L. A. (2006). Adults don't always know best: Preschoolers use past reliability over age when learning new words. Psychological Science, 17, 757-758. doi: 10.1111/j.1467-9280.2006.01778.x

Josephs, M., Kushnir, T., Gräfenhain, M., \& Rakoczy, H. (2016). Children protest moral and conventional violations more when they believe actions are freely chosen. Journal of Experimental Child Psychology, 141, 247-255. doi: 10.1016/j.jecp.2015.08.002

Kallgren, C. A., Reno, R. R., \& Cialdini, R. B. (2000). A focus theory of normative conduct: When norms do and do not affect behavior. Personality and Social Psychology Bulletin, 26, 1002-1012. doi: 0.1177/01461672002610009 
Kapitány, R., \& Nielsen, M. (2015). Adopting the ritual stance: The role of opacity and context in ritual and everyday actions. Cognition, 145, 13-29. doi: doi:10.1016/j.cognition.2015.08.002

Kenward, B., Karlsson, M., \& Persson, J. (2010). Over-imitation is better explained by norm learning than by distorted causal learning. Proceedings of the Royal Society B: Biological Sciences, 278, 1239-1246. doi: 10.1098/rspb.2010.1399

Keupp, S., Behne, T., \& Rakoczy, H. (2013). Why do children overimitate? Normativity is crucial. Journal of Experimental Child Psychology, 116, 392-406. doi: 10.1016/j.jecp.2013.07.002

Köymen, B., Lieven, E., Engemann, D. A., Rakoczy, H., Warneken, F., \& Tomasello, M. (2014). Children's norm enforcement in their interactions with peers. Child Development, 85(3), 1108-1122. doi: 10.1111/cdev.12178

Lancy, D. F. (2010). Learning 'from nobody': The limited role of teaching in folk models of children's development. Childhood in the Past, 3(1), 79-106.

Lancy, D.F. (2015). The Anthropology of Childhood: Cherubs, Chattel, \& Changelings (2nd ed.). Cambridge, U.K.: Cambridge University Press.

Lancy, D. F. (2016). Playing With Knives: The Socialization of Self-Initiated Learners. Child Development, 87, 654-665. doi:10.1111/cdev.12498

Landis, J. R., \& Koch, G. G. (1977). The measurement of observer agreement for categorical data. Biometrics, 159-174. doi: 10.2307/2529310

Lave, J., \& Wenger, E. (1991). Situated learning: Legitimate peripheral participation. Cambridge: Cambridge University Press. 
Legare, C.H. \& Harris, P.L. (2016). The ontogeny of cultural learning. Child Development, 87(3), 633-642. doi: 10.1111/cdev.12542

Legare, C. H., \& Nielsen, M. (2015). Imitation and innovation: the dual engines of cultural learning. Trends in Cognitive Sciences, 19(11), 688-699. doi: 10.1016/j.tics.2015.08.005

Legare, C. H. \& Watson-Jones, R. E. (2015). The evolution and ontogeny of ritual. In D. M. Buss (Ed.), The Handbook of Evolutionary Psychology. Hoboken, NJ: Wiley \& Sons.

Legare, C.H., Wen, N.J., Herrmann, P.A., \& Whitehouse, H. (2015). Imitative flexibility and the development of cultural learning. Cognition, 142, 351-361. doi: 10.1016/j.cognition.2015.05.020

Little, E.E., Carver, L.J., \& Legare, C.H. (2016). Cultural variation in triadic infant-caregiver object exploration. Child Development, 87(4), 1130-1145. doi: 10.1111/cdev.12513

López, A., Correa-Chávez, M., Rogoff, B., \& Gutiérrez, K. (2010). Attention to instruction directed to another by US Mexican-heritage children of varying cultural backgrounds. Developmental Psychology, 46, 593-601. doi: 10.1037/a0018157

Lyons, D. E., Damrosch, D. H., Lin, J. K., Macris, D. M., \& Keil, F. C. (2011). The scope and limits of overimitation in the transmission of artefact culture. Philosophical Transactions of the Royal Society of London B: Biological Sciences, 366(1567), 1158-1167. doi: 10.1098/rstb.2010.0335

Lyons, D.E, Young, A.G. \& Keil, F.C. (2007). The hidden structure of overimitation. Proceedings of the National Academy of Sciences, 104, 19751-19756. doi: 10.1073/pnas.0704452104 
McGuigan, N., Whiten, A., Flynn, E., \& Horner, V. (2007). Imitation of causally opaque versus causally transparent tool use by 3-and 5-year-old children. Cognitive Development, 22, 353-364. doi: $10.1098 /$ rstb.2009.0069

Nielsen, M. (2006). Copying actions and copying outcomes: social learning through the second year. Developmental Psychology, 42, 555. doi: 10.1037/0012-1649.42.3.555

Nielsen, M., \& Blank, C. (2011). Imitation in young children: When who gets copied is more important than what gets copied. Developmental Psychology, 47(4), 1050-1053. doi: $10.1037 / \mathrm{a} 0023866$

Nielsen, M., Cucchiaro, J., \& Mohamedally, J. (2012). When the transmission of culture is child's play. PloS one, 7, e34066. doi: 10.1371/journal.pone.0034066

Nielsen, M., Kapitány, R., \& Elkins, R. (2015). The perpetuation of ritualistic actions as revealed by young children's transmission of normative behavior. Evolution and Human Behavior, 36(3), 191-198. doi:10.1016/j.evolhumbehav.2014.11.002

Nielsen, M., Mushin, I., Tomaselli, K. \& Whiten, A. (2016). Imitation, collaboration, and their interaction among Western and Indigenous Australian preschool children. Child Development, 87, 795-806. doi:10.1111/cdev.12504

Nielsen, M., Simcock, G., \& Jenkins, L. (2008). The effect of social engagement on 24-montholds' imitation from live and televised models. Developmental Science, 11(5), 722-731. doi: $10.1111 / \mathrm{j} .1467-7687.2008 .00722 . x$

Over, H., \& Carpenter, M. (2009). Priming third-party ostracism increases affiliative imitation in children. Developmental Science, 12, F1-F8. doi: 10.1111/j.1467-7687.2008.00820.x 
Over, H., \& Carpenter, M. (2012). Putting the social into social learning: Explaining both selectivity and fidelity in children's copying behavior. Journal of Comparative Psychology, 126, 182. doi: 10.1037/a0024555

Over, H., \& Carpenter, M. (2013). The social side of imitation. Child Development Perspectives, 7, 6-11. doi: 10.1111/cdep.12006

Pérez-Grandos, D.R., \& Callanan, M.A. (1997). Conversations with mothers and siblings: Young children's semantic and conceptual development. Developmental Psychology, 33, 120134. doi: $10.1037 / 0012-1649.33 .1 .120$

Radziszewska, B., \& Rogoff, B. (1989). Influence of adult and peer collaborators on children's planning skills. Developmental Psychology, 24, 840-848. doi: 10.1037/0012-1649.24.6.840

Radziszewska, B., \& Rogoff, B. (1991). Children's guided participation in planning imaginary errands with skilled adult of peer partners. Developmental Psychology, 27, 381-389. doi: $10.1037 / 0012-1649.27 .3 .381$

Rakoczy, H., \& Schmidt, M. F. H. (2013). The early ontogeny of social norms. Child Development Perspectives, 7(1), 17-21. doi: 10.1111/cdep.12010

Rakoczy, H., Warneken, F., \& Tomasello, M. (2008). The sources of normativity: Young children's awareness of the normative structure of games. Developmental Psychology, 44, 875-881. doi: 10.1037/0012-1649.44.3.875

Rogoff, B. (2003). The cultural nature of human development. New York: Oxford University Press. 
Rogoff, B., Ellis, S., \& Gardner, W. (1984). Adjustment of adult-child instruction according to child's age and task. Developmental Psychology, 20, 103-199. doi: 10.1037/0012-1649.20.2.193

Schachner, A., \& Carey, S. (2013). Reasoning about 'irrational' actions: When intentional movements cannot be explained, the movements themselves are seen as the goal. Cognition, 129, 309-327. doi: 10.1016/j.cognition.2013.07.006

Subiaul, F., Patterson, E. M., Schilder, B., Renner, E., \& Barr, R. (2014). Becoming a high-fidelity-super-imitator: what are the contributions of social and individual learning? Developmental Science, 18(6), 1025-1035. doi: 10.1111/desc.12276

Subiaul, F., \& Schilder, B. (2014). Working memory constraints on imitation and emulation. Journal of Experimental Child Psychology, 128, 190-200. doi: 10.1016/j.jecp.2014.07.005

VanderBorght, M. \& Jaswal, V.K. (2009). Who knows best? Preschoolers sometimes prefer child informants over adult informants. Infant \& Child Development, 18(1), 61-71. doi: 10.1002/icd.591

Watson-Jones, R.E. \& Legare, C.H. (2016). The social functions of group rituals. Current Directions in Psychological Science, 25, 42-46. doi: 10.1177/0963721415618486

Watson-Jones, R.E., Legare, C.H., Whitehouse, H., \& Clegg, J.M. (2014). Task-specific effects of ostracism on imitative fidelity in early childhood. Evolution and Human Behavior, 35, 204-210. doi:10.1016/j.evolhumbehav.2014.01.004

Watson-Jones, R. E., Whitehouse, H., \& Legare, C. H. (2016). In-group ostracism increases high-fidelity imitation in early childhood. Psychological Science, 27(1), 34-42. doi: $10.1177 / 0956797615607205$ 
Wen, N. J., Herrmann, P. A., \& Legare, C. H. (2016). Ritual increases children's affiliation with in-group members. Evolution and Human Behavior, 37(1), 54-60. doi: 10.1016/j.evolhumbehav.2015.08.002

Yu, Y., \& Kushnir, T. (2013). Social context effects in 2-and 4-year-olds' selective versus faithful imitation. Developmental Psychology, 50(3), 922. doi: 10.1037/a0034242 
Table A1.

Summary of multiple regression analysis for imitative fidelity scores.

\begin{tabular}{lccccc}
\hline & $\mathrm{B}$ & $\mathrm{SE}(\mathrm{B})$ & $\beta$ & $t$ & $p$ \\
\cline { 2 - 6 } Condition & 0.90 & 0.30 & 0.34 & 2.99 & .004 \\
Child age & 0.28 & 0.14 & 0.23 & 2.03 & .047 \\
\hline
\end{tabular}

Table A2.

Summary of multiple regression analysis for instructed imitative fidelity.

\begin{tabular}{lccccc}
\hline & $\mathrm{B}$ & $\mathrm{SE}(\mathrm{B})$ & $\beta$ & $t$ & $p$ \\
\cline { 2 - 6 } Condition & 0.12 & 0.05 & 0.25 & 2.19 & .032 \\
Child age & 0.05 & 0.03 & 0.24 & 2.07 & .042 \\
\hline
\end{tabular}

